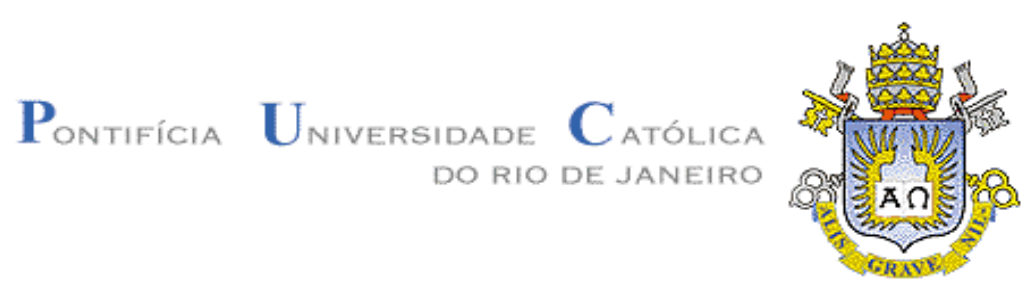

Flávia Cristina da Costa Serrão

\title{
Modelo de Previsão de Carga de Curto Prazo Utilizando Redes Neurais e Lógica Fuzzy
}

\begin{abstract}
Dissertação de Mestrado
Dissertação apresentada como requisito parcial para obtenção do grau de Mestre pelo Programa de Pósgraduação em Engenharia Elétrica do Departamento de Engenharia Elétrica da PUC-Rio.
\end{abstract}

Orientador: Prof. Reinaldo Castro Souza

Rio de Janeiro

Março de 2003 


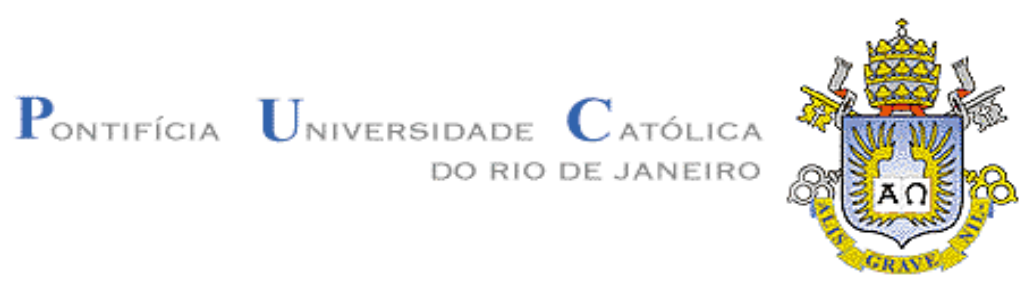

Flávia Cristina da Costa Serrão

\title{
Modelo de Previsão de Carga de Curto Prazo Utilizando Redes Neurais e Lógica Fuzzy
}

\begin{abstract}
Dissertação apresentada como requisito parcial para obtenção do grau de Mestre pelo Programa de Pós-graduação em Engenharia Elétrica do Departamento de Engenharia Elétrica do Centro Técnico Científico da PUC-Rio. Aprovada pela Comissão Examinadora abaixo assinada.
\end{abstract}

\author{
Prof. Reinaldo Castro Souza \\ Orientador \\ Departamento de Engenharia Elétrica - PUC-Rio \\ Prof. Gutemberg Hespanha Brasil \\ Departamento de Estatística - UFES \\ Prof. João Carlos Aires \\ Instituto de Energia - PUC-Rio/Light \\ Prof. Tufi Machado Soares \\ Departamento de Estatística - UFJF \\ Prof. Ney Dumont \\ Coordenador Setorial do Centro Técnico Científico - Puc-Rio
}


Todos os direitos reservados. É proibida a reprodução total ou parcial do trabalho sem autorização da universidade, da autora e do orientador.

Flávia Cristina da Costa Serrão Graduou-se em Estatística na UERJ (Universidade do Estado do Rio de Janeiro) em 1999.

Ficha Catalográfica

Modelo de previsão de carga de curto prazo utilizando redes neurais e lógica fuzzy / Flávia Cristina da Costa Serrão; orientador: Reinaldo Castro Souza. - Rio de Janeiro : PUC, Departamento de Engenharia Elétrica, 2003.

[13], 98 f. : il. ; $30 \mathrm{~cm}$

Dissertação (mestrado) - Pontifícia Universidade Católica do Rio de Janeiro, Departamento de Engenharia Elétrica.

Inclui referências bibliográficas.

1. Engenharia elétrica - Teses. 2. Previsão de carga de curto prazo. 3. Redes neurais. 4. Lógica fuzzy. I. Souza, Reinaldo Castro. II. Pontifícia Universidade Católica do Rio de Janeiro. Departamento de Engenharia Elétrica. III. Título. 
Dedico esta dissertação ao meu marido Márcio Lessa, aos meus pais Lacir e Getulio Serrão, a minha avó Eluzia. 


\section{Agradecimentos}

A Deus por me alimentar todos os dias com sua força.

Ao meu orientador Prof. Reinaldo Castro Souza por acreditar e apostar em mim.

Ao CNPq e a PUC-Rio, pelos auxílios concedidos.

Ao meu marido Márcio Luiz Lessa por todo seu amor, apoio e paciência. Por estar sempre ao meu lado e fazer com que eu acreditasse que era possível.

Aos meus pais Lacir e Getulio por me ensinarem a viver, me dando um amor incondicional e sendo sempre exemplos de dignidade, honestidade e perseverança.

A minha avó Eluzia por seu amor sem limites e suas orações.

Aos meus sobrinhos pelo simples fato de existirem e serem razão de minha vida e as minhas irmãs Márcia e Ana Paula por seu amor, amizade e incentivo.

A todas as pessoas de minha família e amigos pela torcida.

As minhas amigas de todas as horas Cristiane Camacho e Rosane Kirchner por estarem sempre ao meu lado, chorando ou sorrindo, como dizem por aí "...se choramos ou se sorrimos o importante é que emoções nós vivemos...”

Ao pessoal do LEC, aos meninos do NEC, ao Carlinhos, a Ana Paiva e Flávio, ao pessoal da secretaria do DEE Alcina, Ana, Márcia e Danilo, aos meninos da sala 442 em especial Evandro e Isnarde (a culpa é sempre dele!!), Luiz Fernando (LF). Agradecer por todo o carinho e atenção dispensados a mim.

A Ana Paula, Lucio, Eliane, Rodrigo, Felipe (Felipão), Kelly, Janaína, Mayte, Luiz Camacho por sua amizade e ajuda sempre na hora certa.

Ao Sr Plutarcho Maravilha Lourenço por suas sugestões e ajuda.

Aos professores que participaram da Comissão Examinadora. 


\section{Resumo}

Serrão, Flávia Cristina da Costa; Souza, Reinaldo Castro. Modelo de Previsão de Carga de Curto Prazo Utilizando Redes Neurais e Lógica Fuzzy. Rio de Janeiro, 2003. 111p. Dissertação de Mestrado - Departamento de Engenharia Elétrica, Pontifícia Universidade Católica do Rio de Janeiro.

O objetivo principal desta dissertação é desenvolver um método de previsão de carga elétrica de curto prazo (previsão horária), através de um sistema híbrido (Redes Neurais e Lógica Fuzzy) utilizando temperaturas máximas e mínimas como variáveis explicativas. Como primeiro passo, foram definidos os perfis homogêneos das curvas de carga diárias através de um classificador utilizando os Mapas Auto Organizáveis (Self-Organizing Maps-SOM). Um previsor será adicionado ao esquema de previsão através da Lógica Fuzzy que associará as variáveis climáticas aos perfis criados pela SOM produzindo as previsões.

O modelo foi aplicado em dados de duas concessionárias de energia elétrica do Brasil usando dados horários coletados durante dois anos.

\section{Palavras-chave}

Previsão de carga de curto prazo; Redes Neurais; Lógica Fuzzy. 


\section{Abstract}

Serrão, Flávia Cristina da Costa; Souza, Reinaldo Castro (Advisor). A shortterm load forecasting model using Neural Network and Fuzzy Logic. Rio de Janeiro, 2003. 111p. MSc. Dissertation - Departamento de Engenharia Elétrica, Pontifícia Universidade Católica do Rio de Janeiro.

This dissertation presents a short-term load forecasting procedure mixing a classifier scheme and a predictive scheme. The classifier is implemented through an artificial neural network using a non-supervised learning procedure (SOM). Concerning the predictive scheme, a fuzzy logic procedure uses climatic variables and their prediction to choose the appropriate profiles created by SOM and then combines them to produce the desired forecast.

The model is applied to two utilities in Brazil using hourly observations collected during two calendar years and the results obtained, in terms of mean absolute percentage error (MAPE) through the period analyzed, are presented.

\section{Keywords}

Short-term load forecasting; Neural networks; Logic Fuzzy 


\section{Sumário}

1 Introdução 14

1.1. Motivação 14

1.2. Objetivo 16

$\begin{array}{ll}\text { 1.3. Organização do texto } & 18\end{array}$

2 As concessionárias $\quad 20$

2.1. ENERSUL. 20

2.2. ESCELSA 21

3 Mercado de Energia Elétrica 22

4 Curvas de carga $\quad 25$

4.1. Variáveis meteorológicas para previsão de carga 26

4.2. Modelagem da relação Carga x Temperatura 28

5 Inteligência Computacional 31

5.1. Lógica Fuzzy

5.1.1. Introdução 32

5.1.2. Conceitos básicos 33

5.1.3. Conjuntos fuzzy (nebulosos) 34

5.1.4. Operações entre conjuntos fuzzy 37

5.1.5. Sistema Fuzzy 37

5.2. Redes Neurais 42

5.2.1. Aprendizado Supervisionado $\quad 47$

5.2.2. Aprendizado Não Supervisionado 48

6 Modelo Proposto $\quad 51$

6.1. Introdução 51

6.2. Os dados disponíveis 51

6.3. O modelo 53 
7 Aplicação do modelo com dados reais $\quad 58$

$\begin{array}{ll}\text { 7.1. Análises dos resultados } & 60\end{array}$

8 Conclusões e trabalhos futuros $\quad 68$

$\begin{array}{ll}\text { 8.1. Descrição do algoritmo } & 70\end{array}$

9 Referências bibliográficas $\quad 73$

$\begin{array}{ll}\text { Anexo } 1 & 76\end{array}$

$\begin{array}{lr}\text { Anexo } 2 & 81\end{array}$

$\begin{array}{ll}\text { Anexo } 3 & 85\end{array}$ 


\section{Lista de figuras}

Figura 1 - Área de concessão da ENERSUL 20

Figura 2 - Área de concessão da ESCELSA 21

Figura 3 - Curvas de carga dos dias 01 e 08/10/2002 26

Figura 4 - Curvas de carga dos dias 16 e 23/10/2002 27

Figura 5 - Cargas x Temperatura horárias dos EUA 28

Figura 6 - Carga de pico e temperatura máximas Tóquio 29

Figura 7 - Carga x Temperatura horária ENERSUL 29

Figura 8 - Diagrama de dispersão ENERSUL 30

Figura 9 - Sistema Fuzzy 33

Figura 10 - Conjuntos fuzzy $\quad 36$

Figura 11 - Saída sistema inferência Mandani 38

Figura 12 - Saída sistema inferência Takagi-Sugeno 39

Figura 13 - Saída sistema inferência Tsukamoto 40

Figura 14 - Neurônio biológico e artificial 43

Figura 15 - Rede neural direta 45

Figura 16 - Modelo básico de um neurônio 46

Figura 17 - Procedimento treinamento supervisionado 47

Figura 18 - Procedimento treinamento não supervisionado 49

Figura 19 - Rede de Kohonen $\quad 50$

Figura 20 - Cargas média diárias de 2002 ESCELSA 52

Figura 21 - Cargas médias diárias de 2002 ENERSUL 52

Figura 22 - Esquema do procedimento proposto 54

Figura 23 - Conjuntos Fuzzy para temperatura mínima 59

Figura 24 - Conjuntos Fuzzy para temperatura máxima 59

Figura 25 - Saída do sistema para o dia 15/02/2001 60

Figura 26 - Saída do sistema para o dia 03/10/2001 60

Figura 27 - Evolução da carga média horária dos últimos quatro anos do mês de outubro. $\quad 64$

Figura 28 - Evolução da carga média horária dos últimos quatro anos do mês de outubro. 
Figura 29 - Carga média A2 outubro de $2002 \quad 65$

Figura 30 - Carga média A2 novembro de 2002

Figura 31 - Gráfico de freqüência com as quantidades mínimas de carga A2 outubro de 2002

Figura 32 - Gráfico de freqüência com as quantidades mínimas de carga A2 novembro de $2002 \quad 67$

Figura 33 - Exemplo de formulação das regras fuzzy 71

Figura 34 - Primeira ilustração para exemplo do Algoritmo da SOM. $\quad 81$

Figura 35- Segunda ilustração para exemplo do Algoritmo da SOM. 82

Figura 36 - Terceira ilustração para exemplo do Algoritmo da SOM. 83

Figura 37 - Quarta ilustração para exemplo do Algoritmo da SOM. 84 


\section{Lista de tabelas}

Tabela 1 - Vantagens e desvantagens da Lógica Fuzzy 41

Tabela 2 - classes (neurônios) 58

Tabela 3 - tabelas com estatísticas de erros do modelo 62

Tabela 4 - MAPE 63

Tabela 5 - MAPEP 63

Tabela 6 - MAPEF $\quad 63$

Tabela 7 - Tabela para formulação das regras fuzzy 72 
"Litlle people discuss other people. Medium people discuss events.

Big people discuss ideas."

R. E. Kalman, 1962 